\title{
Dietary intake during a Royal Navy submarine deployment
}

\author{
F. Gunner ${ }^{1}$, M. Lindsay ${ }^{1}$, P.E.H. Brown ${ }^{1}$, A.M. Shaw ${ }^{1}$, S.E. Britland ${ }^{1}$, T. Davey ${ }^{1}$, \\ S.A. Lanham-New ${ }^{2}$, B.A. Griffin ${ }^{2}$ and J.L. Fallowfield ${ }^{1}$ \\ ${ }^{1}$ Environmental and Medicine Sciences, The Institute of Naval Medicine, Gosport, Hampshire, UK and \\ ${ }^{2}$ Department of Nutritional Sciences, School of Biosciences and Medicine, Faculty of Health and Medical Sciences, \\ University of Surrey, Guildford, Surrey, UK
}

Royal Navy (RN) submarines present a unique occupational setting that exposes individuals to confined space, a lack of natural light, disruptive working patterns, and limited opportunities for physical exercise. Restricted storage space and prolonged submersion also limits access to a variety of fresh food, which imposes a challenge to achieving an adequate total energy intake, and a nutritionally balanced intake to maintain health. Information on the dietary habits of operationally deployed submariners is limited, therefore this study aimed to monitor the dietary intake of RN submariners whilst deployed.

Two cohorts of male submariners on separate operational deployments (SUB1: $n=36$; SUB2: $n=60$ ) completed a 4-day food diary early in the deployment (DIET 1) and within 2 weeks prior to the end of deployment (DIET 2). The deployment period at sea was 3 months, during which food provision was not replenished. Dietary intake data were analysed using a dietary assessment software package (DietPlan 6). The study was approved by the Ministry of Defence Research Ethics Committee (Ref: 0903/228) and volunteers provided informed consent. Data were tested for normality (Shapiro-Wilk test) before differences between early and late stage intakes were assessed using a paired sample t-test.

There were no significant differences in the mean intakes of total energy and macronutrients across the deployment for either submarine cohort (Table 1).

\begin{tabular}{|c|c|c|c|c|c|c|c|c|}
\hline & \multicolumn{4}{|l|}{ SUB 1} & \multicolumn{4}{|l|}{ SUB 2} \\
\hline & DIET 1 & SD & DIET 2 & SD & DIET 1 & SD & DIET 2 & $\mathrm{SD}$ \\
\hline Energy Intake (kJ) & 10494 & 2569 & 10699 & 2824 & 9494 & 2297 & 9025 & 2197 \\
\hline Protein ( \%) & 17 & 3 & 18 & 2 & 20 & 8 & 19 & 6 \\
\hline Carbohydrate (\%) & 43 & 6 & 41 & 4 & 42 & 8 & 44 & 6 \\
\hline Fat $(\%)$ & 40 & 6 & 40 & 5 & 37 & 5 & 37 & 4 \\
\hline Saturated fat ( \%) & 14 & 3 & 14 & 3 & 12 & 2 & 11 & 2 \\
\hline
\end{tabular}

In conclusion, dietary intake of RN submariners was not different during the early and late stages of a 3-month submarine deployment. Whilst total energy intake was maintained, despite potential limitations from a provision perspective, it was lower than public health guidelines. ${ }^{1}$ Nevertheless, data are consistent with some ${ }^{2}$ but not all ${ }^{3}$ previously reported literature in submariner cohorts. Importantly, the energy requirement of the submariner occupational role has not been defined, therefore future work is required to determine energy expenditure to better inform dietary provision.

1. Scientific Advisory Committee for Nutrition Dietary Reference Values for Energy (2011).

2. Gasier H.G. et al., (2016) Nutrients 8(2):E85.

3. Singh V.K. et al., (2011) Defence Science Journal 61(6):540-544. 